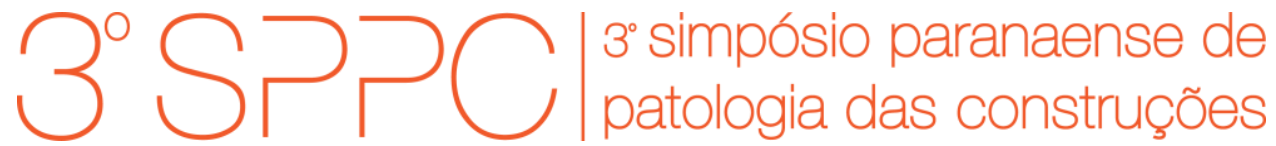

ISSN 2526-7248 artigo n. 3SPPC1007, pp. 72-83, 2018

\title{
Proposta de modelo de análise da vulnerabilidade da operação de pontes e viadutos
}

\author{
Domingues, Gabriela Becker ${ }^{1}$; Santos Filho, Mauro Lacerda ${ }^{2}$; Parchen, Carlos \\ Frederico Alice $^{3}$ \\ ${ }^{1}$ Mestranda, Universidade Federal do Paraná, gabrielabdomingues@gmail.com \\ 2 Professor, Universidade Federal do Paraná, maurolacerda1982@gmail.com \\ ${ }^{3}$ Professor, Universidade Federal do Paraná, parchen.ufpr@gmail.com
}

\begin{abstract}
Resumo: Pontes e viadutos são obras de infraestrutura essenciais para o desenvolvimento social e econômico do território, e assim sistemas de gestão de obras de arte especiais (OAEs) são estudados em inúmeros países. O presente trabalho propõe um modelo de análise da vulnerabilidade da operação de OAEs, que indica quais são os elementos da obra com maior urgência de intervenção, e permite a simulação de cenários de deterioração, auxiliando na tomada de decisão sobre a destinação de recursos financeiros para manutenção. A vulnerabilidade é a relação entre o dano e a sua consequência para a operação da estrutura: quanto menor for o esforço necessário para provocar um dano e maior for a consequência para a operação, maior será a vulnerabilidade. A ferramenta apresentada foi desenvolvida a partir da Metodologia de Inspeção e Avaliação de OAEs da Universidade Federal do Paraná (UFPR), e estabelece uma analogia à Teoria da Vulnerabilidade Estrutural. O método foi aplicado a uma ponte que integra o grupo de 520 OAEs objeto de estudo em parceria firmada entre a UFPR e o Departamento Nacional de Infraestrutura de Transportes. O modelo proposto fornece ao gestor uma ótica diferenciada, e com aprimoramento matemático e validação dos parâmetros adotados, mostra-se uma ferramenta inovadora e interessante para utilização em um sistema de gestão de OAEs.
\end{abstract}

Palavras-chave: Obras de arte especiais, pontes, gestão, inspeção, vulnerabilidade.

Abstract: Bridges and viaducts are essential infrastructure works for the social and economic development of the territory, so that bridge and viaducts management systems are studied in many countries. The present work proposes a vulnerability analysis model of bridges and viaducts, which indicates the elements with greater urgency of intervention, and allows the simulation of deterioration scenarios, helping in the decision making on the allocation of financial resources for maintenance of these structures. Vulnerability is understood as the relationship between the damage and its consequence for the operation of the structure: the less effort required to cause damage and the greater the consequence for the operation, greater will be the vulnerability. The tool presented was developed from the Bridges and Viaducts Inspection and Evaluation Methodology of the Federal University of Paraná (UFPR), and establishes an analogy to Structural Vulnerability Theory. The method was applied to a bridge which is part of the group of 520 bridges and viaducts under study in partnership between UFPR and the National Department of Transport Infrastructure. The proposed model provides a different perspective to the manager, and with mathematical improvement and validation of the adopted parameters, will be an innovative and interesting tool for use in a bridge and viaduct management system.

Keywords: Bridges, management, inspection, vulnerability. 
DOMINGUES, G. B.; SANTOS FILHO, M. L.; PARCHEN, C. F. A., PROPOSTA DE MODELO DE ANÁLISE DA VULNERABILIDADE DA OPERAÇÃO DE PONTES E VIADUTOS. $3^{\circ}$ Simpósio Paranaense de Patologia das Construções (3 SPPC), artigo 3SPPC1007, pp. $72-83$, 2018. DOI: $10.4322 / 2526-7248.007$

\section{Introdução}

Obras de Arte Especiais (OAEs) possuem papel primordial no desenvolvimento social e econômico do território, seja no âmbito das micro ou macro regiões. Este impacto positivo é acentuado no Brasil, onde os modais rodoviário e ferroviário, compostos por um grande número de pontes e viadutos, são responsáveis pelo transporte de aproximadamente $81,8 \%$ das cargas movimentadas no território [3].

A Confederação Nacional dos Transportes (CNT) [4] divulgou no seu último relatório que de $105.814 \mathrm{~km}$ de rodovias analisadas, apenas $38,2 \%$ tem estado geral classificado como ótimo ou bom. Entre os aspectos avaliados estão as OAEs, presentes em $54.972 \mathrm{~km}$, onde somente 25,7\% possuem barreiras laterais e acostamento completos, $64,2 \%$ não apresentam um destes dispositivos, e em $10,1 \%$ não há nem defensa nem acostamento. A pesquisa também aponta que as obras sob gestão concedida apresentam melhores condições se comparadas às obras sob gestão pública.

Dado este cenário o Governo Brasileiro vem investindo no aprimoramento do seu Sistema de Gestão de OAEs (SGO). Em uma parceria entre o Departamento Nacional de Infraestrutura de Transportes (DNIT) e a Universidade Federal do Paraná (UFPR), através do Escritório Modelo de Engenharia Civil (EMEA), estão sendo desenvolvidas melhorias para o SGO, além do incremento do banco de dados existente através da inspeção de aproximadamente 520 OAEs, localizadas nos estados do Paraná e Santa Catarina.

Um dos estudos em andamento consiste em uma metodologia de análise da vulnerabilidade da operação de OAEs, que objetiva investigar quais as consequências no desempenho de uma obra quando submetida a algum tipo de dano estrutural ou funcional. Deste modo é possível identificar quais reparos são mais eficientes para a melhoria global da operação da ponte, permitindo uma racionalização dos investimentos para revitalização destas obras.

A proposta apresentada neste trabalho destaca-se por permitir a simulação de comportamentos futuros da obra. Outro ponto relevante é o fato de considerar não apenas os aspectos estruturais, mas também de durabilidade dos elementos, e operacionais, que permitem ao usuário uma experiência segura e confortável.

O modelo de Análise da Vulnerabilidade da Operação de Pontes e Viadutos foi desenvolvido estabelecendo uma analogia à Teoria da Vulnerabilidade Estrutural [8] e utilizando parâmetros provenientes do Modelo de Avaliação da Performance de OAEs da UFPR [6,7]. Este artigo apresenta uma breve introdução às fontes de desenvolvimento supracitadas, a metodologia adotada para desenvolvimento do modelo, a apresentação da proposta de modelo de análise da vulnerabilidade da operação de pontes e viadutos, e a aplicação deste em uma ponte.

\section{Referencial teórico}

\subsection{Modelo UFPR de avaliação da performance de obras de arte especiais}

A Universidade Federal do Paraná desenvolveu um modelo de avaliação da performance de OAEs, objetivando apresentar uma metodologia capaz de analisar 
DOMINGUES, G. B.; SANTOS FILHO, M. L.; PARCHEN, C. F. A., PROPOSTA DE MODELO DE ANÁLISE DA VULNERABILIDADE DA OPERAÇÃO DE PONTES E VIADUTOS. $3^{\circ}$ Simpósio Paranaense de Patologia das Construções (3 SPPC), artigo 3SPPC1007, pp. $72-83$, 2018. DOI: $10.4322 / 2526-7248.007$

as condições estruturais e funcionais destas obras, culminando em um ranqueamento segundo a urgência de intervenção. O Modelo UFPR utiliza as informações coletadas nas inspeções de campo, as parametriza, e retrata através de um índice numérico o grau de deterioração da obra, para que esta informação seja usada como critério na determinação da urgência intervenção. As notas de cada obra são relativizadas para permitir a comparação entre diferentes estruturas, viabilizando o seu ranqueamento para priorização da destinação de recursos financeiros para reparo e recuperação.

A metodologia desenvolvida pela UFPR é alimentada por informações de cada um dos danos existentes na obra, caracterizados por cinco parâmetros que compõem o "Valor do Dano", e a somatória do valor do dano de todas as deficiências da OAE resulta no seu "Índice de Performance", como apresentado na equação 1:

$$
\mathrm{IP}=\sum \mathrm{VD}=\sum \mathrm{B}_{\mathrm{i}} \times \mathrm{K}_{1 \mathrm{i}} \times \mathrm{K}_{2 \mathrm{i}} \times \mathrm{K}_{3 \mathrm{i}} \times \mathrm{K}_{4 \mathrm{i}}
$$

Onde:

IP = Índice de Performance da OAE;

$\mathrm{VD}=$ Valor do Dano de cada irregularidade verificada na OAE;

$\mathrm{B}_{\mathrm{i}}=$ valor associado ao tipo de dano i (tabela 1 );

$\mathrm{K}_{1 \mathrm{i}}=$ fator que representa a importância do elemento estrutural afetado (tabela 2);

$\mathrm{K}_{2 \mathrm{i}}=$ fator que indica a intensidade do dano i no elemento (tabela 3);

$\mathrm{K}_{3 \mathrm{i}}=$ fator que retrata a ocorrência do dano i em outros elementos do mesmo tipo (tabela 4);

$\mathrm{K}_{4 \mathrm{i}}=$ fator que enfatiza a urgência da intervenção no dano i (tabela 5).

Tabela 1: Valores de B para alguns dos tipos de danos considerados no modelo [6]

\begin{tabular}{cc}
\hline Dano & B \\
\hline Desalinhamento/deslocamento de elemento estrutural & 2,00 \\
Deformação excessiva de elemento estrutural & 5,00 \\
Exposição das estacas da fundação & 3,00 \\
Fissura estrutural & 3,00 \\
Recalque diferencial & 4,00 \\
Fissura mapeada & 1,00 \\
Indícios de fogo, umidade, ou eflorescência (manchas) & 1,00 \\
Deficiência de concretagem & 2,00 \\
Corrosão da armadura & 4,00 \\
Recalque ou deslizamento do aterro & 3,00 \\
Deficiência na junta de dilatação & 2,00 \\
Desnível no tabuleiro & 3,00 \\
Deficiência no sistema de drenagem & 2,00 \\
Buraco no pavimento & 3,00 \\
Sinalização deficiente & 2,00 \\
Inexistência de passeio em área urbana & 2,00 \\
Barreira danificada ou inexistente & 2,00 \\
\hline
\end{tabular}


DOMINGUES, G. B.; SANTOS FILHO, M. L.; PARCHEN, C. F. A., PROPOSTA DE MODELO DE ANÁLISE DA VULNERABILIDADE DA OPERAÇÃO DE PONTES E VIADUTOS. $3^{\circ}$ Simpósio Paranaense de Patologia das Construções (3 SPPC), artigo 3SPPC1007, pp. $72-83$, 2018. DOI: $10.4322 / 2526-7248.007$

Tabela 2: Valores de K1 para alguns dos tipos de elementos considerados no modelo [6]

\begin{tabular}{cc}
\hline Elemento & K1 \\
\hline Encontro; aterro de acesso; laje de aproximação & 0,20 \\
Viga transversina & 0,30 \\
Laje; viga travessa; pilar; aparelho de apoio & 0,40 \\
Elementos de fundação; muro de contenção & 0,40 \\
Viga principal & 0,60 \\
Barreira; guarda rodas; guarda corpo; sinalização & 0,10 \\
Junta de dilatação; sistema de drenagem & 0,20 \\
Pavimento; calçada (passeio) & 0,20 \\
Operação & 0,20 \\
\hline
\end{tabular}

Tabela 3: Valores de K2 [6]

\begin{tabular}{cc}
\hline Porcentagem da área superficial do elemento afetada pelo dano & K2 \\
\hline Menos de 3\% (dano insignificante) & 0,00 \\
De 3\% a 10\% (dano pequeno) & 0,50 \\
De 11\% a 25\% (dano médio) & 1,00 \\
De 26\% a 75\% (dano grande) & 1,50 \\
Mais de 75\% (dano imenso) & 2,00 \\
\hline
\end{tabular}

Tabela 4: Valores de K3 [6]

\begin{tabular}{cc}
\hline Repetição do dano em elementos do mesmo tipo & K3 \\
\hline Aparece em menos de $10 \%$ dos elementos & 0,50 \\
Aparece em 10\% a 25\% dos elementos & 1,00 \\
Aparece em $26 \%$ a $75 \%$ dos elementos & 1,50 \\
Aparece em mais de $75 \%$ dos elementos & 2,00 \\
\hline
\end{tabular}

Tabela 5: Valores de K4 [6]

\begin{tabular}{cc}
\hline Urgência de intervenão no dano & K4 \\
\hline Intervenção não urgente & 1,00 \\
Dano à reparar em até 5 anos & 2,50 \\
Reparo imediato & 4,00 \\
Limitação de carga e interrupção do tráfego & 5,00 \\
\hline
\end{tabular}

O índice de performance deve ser relativizado para que seja possível a comparação dos índices de diferentes OAEs. A relativização é obtida através da divisão pelo "Valor do Dano de Referência", dado pela consideração da ocorrência de todos os danos ("B") que podem afetar os elementos ("K1") que compõem a ponte em análise, multiplicados pelos valores máximos dos fatores de intensidade e extensão do dano ("K2" e "K3"), e pelo valor mínimo para o fator de urgência de reparo ("K4").

Este modelo atualmente está na fase de calibração, e para tal foi aplicado em aproximadamente 520 OAEs situadas nos estados do Paraná e Santa Catarina, através de parceria entre UFPR e DNIT. 
DOMINGUES, G. B.; SANTOS FILHO, M. L.; PARCHEN, C. F. A., PROPOSTA DE MODELO DE ANÁLISE DA VULNERABILIDADE DA OPERAÇÃO DE PONTES E VIADUTOS. $3^{\circ}$ Simpósio Paranaense de Patologia das Construções (30 SPPC), artigo 3SPPC1007, pp. $72-83$, 2018. DOI: 10.4322/2526-7248.007

\subsection{Teoria da Vulnerabilidade de Sistemas Estruturais}

A Teoria da Vulnerabilidade Estrutural (TVE) permite a identificação dos elementos mais vulneráveis de uma estrutura, através do estudo de cenários de falha. Uma estrutura, ou parte dela, é considerada vulnerável se um dano relativamente pequeno causar consequências desproporcionalmente grandes. Por exemplo, se a remoção de uma única viga provocar o colapso imediato de um edifício por completo, entende-se que este elemento apresenta significativa vulnerabilidade [8]. A singularidade da Teoria está no fato de que ela não aponta os eventos de deterioração com maior probabilidade de ocorrência, mas sim os que podem ter graves consequências.

A TVE parte da verificação da forma da estrutura, analisando de que modo os elementos estruturais são conectados uns aos outros, e segundo alguns critérios estes são agrupados em anéis e esferas estruturais. A identificação de cenários de falha inicia pelo processo de agrupamento, quando os anéis e esferas formam uma descrição hierárquica da estrutura, e pelo processo de descompactação, quando são pesquisados cenários de falha interessantes ao longo da hierarquia estabelecida [8].

Escolhido um cenário de falha para estudo, deve-se calcular o seu "Índice de Vulnerabilidade" através da razão entre sua "Consequência" e sua "Demanda de Dano Relativa", conforme a equação 2:

$$
I=\frac{C}{D_{R}}
$$

Onde:

I = Índice de Vulnerabilidade do cenário de falha;

$\mathrm{C}=$ Consequência do cenário de falha;

$\mathrm{DR}=$ Demanda de Dano Relativa para ocorrência do cenário.

A consequência que um cenário de falha tem em uma estrutura é mensurada pela razão entre o decréscimo na qualidade de formação que a estrutura teve decorrente da sua deterioração, e a qualidade de formação da estrutura intacta [8], como demonstra a equação 3 :

$$
C=\frac{Q_{\text {intacta }}-Q_{\text {deteriorada }}}{Q_{\text {intacta }}}
$$

Onde:

$Q_{\text {intacta }}=$ Qualidade de Formação da estrutura sem dano;

$Q_{\text {deteriorada }}=$ Qualidade de Formação da estrutura após o dano.

O esforço necessário para causar um evento de deterioração é diretamente proporcional à perda de rigidez do elemento afetado por tal evento, essa quantificação é a "Demanda de Dano". A "Máxima Demanda de Dano" ocorre quando todos os membros do cenário de falha são danificados, e a razão entre a demanda de dano de um cenário de falha e demanda de dano máxima para esse mesmo cenário, é a "Demanda de Dano Relativa", como mostra a equação 4: 


$$
D_{R}=\frac{D}{D_{\text {máx }}}
$$

Onde:

$\mathrm{D}=$ Demanda de Dano do cenário de falha;

$D_{\text {máx }}=$ Demanda de Dano Máxima para o cenário de falha.

\section{Metodologia}

A presente proposta de análise da vulnerabilidade da operação de OAEs foi desenvolvida estabelecendo uma analogia à Teoria da Vulnerabilidade Estrutural, e utilizando como dados de entrada os parâmetros provenientes do Modelo UFPR. Para tal foram estudadas diferentes alternativas e neste trabalho é apresentado 0 método que avalia todos os elementos e sistemas que podem constituir uma OAE.

As etapas de desenvolvimento foram:

1. Identificação dos principais componentes de uma OAE e agrupamento destes, segundo suas similaridades, em "subsistemas de operação";

2. Definição dos parâmetros do Modelo UFPR para caracterização e avaliação cada componente;

3. Criação de uma estrutura hipotética de representação dos subsistemas da OAE (para viabilizar a aplicação dos conceitos da TVE); e estudo das principais interações entre os subsistemas de operação pra definição da posição ocupada por cada um na estrutura hipotética;

4. Definição dos critérios de agrupamento para composição da estrutura hierárquica;

5. Definição do cálculo do modelo;

6. Simulação de cenários de falha para teste da proposta elaborada.

\section{Resultados}

\subsection{Modelo de Análise da Vulnerabilidade da Operação de Pontes e Viadutos}

O modelo de análise da vulnerabilidade da operação de OAEs, de modo geral, investiga todos os sistemas e elementos que constituem uma OAE, caracterizandoos segundo os principais problemas que podem apresentar, e considerando as interações existentes entre estes sistemas ao longo da vida útil da obra. O processo de análise tem cinco etapas básicas:

1. Caracterização dos subsistemas de operação através dos dados obtidos na inspeção de campo;

2. Cálculo do "Valor do Dano" para os tipos de deterioração verificados, e do "Índice de Performance" dos subsistemas; 
DOMINGUES, G. B.; SANTOS FILHO, M. L.; PARCHEN, C. F. A., PROPOSTA DE MODELO DE ANÁLISE DA VULNERABILIDADE DA OPERAÇÃO DE PONTES E VIADUTOS. $3^{\circ}$ Simpósio Paranaense de Patologia das Construções (30 SPPC), artigo 3SPPC1007, pp. $72-83$, 2018. DOI: $10.4322 / 2526-7248.007$

3. Agrupamento dos subsistemas segundo os critérios estabelecidos, resultando no diagrama de descrição hierárquica da ponte;

4. Simulação de danos para investigação dos cenários de falha;

5. Cálculo da "Consequência Estrutural", da "Demanda de Dano Relativa", e do "Índice de Vulnerabilidade" para os cenários desejados.

\subsubsection{Subsistemas de Operação}

A análise da operacionalidade de uma ponte requer a observação de todos os fatores que possibilitam o desempenho adequado da mesma, envolvendo desde os elementos estruturais até os elementos e sistemas complementares. Assim, foram definidos doze grupos, nomeados "Subsistemas de Operação": desempenho dos materiais, defeitos construtivos, desempenho estrutural, fundações e aterros, juntas de movimentação e aparelhos de apoio, drenagem, pavimento, segurança, conforto do usuário, uso/carregamento estrutural, características hidrológicas e geológicas, características climáticas.

Cada subsistema de operação é representado por quatro tipos de dano característicos. Na tabela 6 é possível observar os danos característicos para alguns dos subsistemas e os respectivos valores de $\mathrm{K} 1$ e $\mathrm{B}$.

Tabela 6: Subsistemas de operação e danos característicos

\begin{tabular}{ccc}
\hline $\begin{array}{c}\text { Subsistema de } \\
\text { operação }\end{array}$ & Dano & B \\
\hline $\begin{array}{c}\text { Desempenho dos } \\
\text { materiais }\end{array}$ & Fissura mapeada & 1,00 \\
$\left(\mathrm{~K}_{1}=0,50\right)$ & Corrosão de armadura & 3,50 \\
& Mancha de umidade ou fogo & 1,00 \\
Defeitos construtivos & Eflorescência & 1,00 \\
$\left(\mathrm{~K}_{1}=0,40\right)$ & Deficiência de cobrimento da armadura & 2,00 \\
& Deficiência de concretagem & 2,00 \\
& Desalinhamento de elemento estrutural & 2,00 \\
Drenagem & Forma aderida ao concreto & 1,00 \\
$\left(\mathrm{~K}_{1}=0,20\right)$ & Inexistência de dreno & 2,00 \\
& Entupimento de dreno & 2,00 \\
Segurança & Quantidade ou tamanho insuficiente & 2,00 \\
$\left(\mathrm{~K}_{1}=0,10\right)$ & Deficiência nas pingadeiras e buzinotes & 2,00 \\
\hline & Sinalização deficiente & 2,00 \\
& Barreira não padronizada & 2,00 \\
Conforto do usuário & Transição perigosa (sem acostamento) & 2,00 \\
$\left(\mathrm{~K}_{1}=0,20\right)$ & Guarda-corpo danificado & 2,00 \\
\hline & Iluminação deficiente & 1,00 \\
& Ausência de passeio em área urbana & 2,00 \\
& Indício de choque de veículos & 1,50 \\
& Nível de vibração elevado & 2,00 \\
\hline
\end{tabular}

Para cada dano que caracteriza um subsistema, é calculado o seu "Valor do Dano" e o "Índice de Performance" do subsistema, conforme o Modelo UFPR (equação 1) 
DOMINGUES, G. B.; SANTOS FILHO, M. L.; PARCHEN, C. F. A., PROPOSTA DE MODELO DE ANÁLISE DA VULNERABILIDADE DA OPERAÇÃO DE PONTES E VIADUTOS. $3^{\circ}$ Simpósio Paranaense de Patologia das Construções (30 SPPC), artigo 3SPPC1007, pp. $72-83$, 2018. DOI: $10.4322 / 2526-7248.007$

e valores de $\mathrm{K} 1$ e $\mathrm{B}$ da tabela 6 . Os valores de $\mathrm{K} 2, \mathrm{~K} 3$ e $\mathrm{K} 4$ são os mesmo do Modelo UFPR (tabelas 3, 4 e 5 respectivamente).

Para permitir uma analogia à TVE foi necessário determinar uma configuração gráfica que retratasse quais subsistemas intergem e/ou influenciam mais uns aos outros durante o funcionamento da obra. A estrutura adotada foi o octaedro, que possui doze arestas, comportando assim os doze subsistemas. Outra característica desta configuração geométrica determinante para sua adoção foi o fato de que todos os vértices são formados por quatro arestas, e assim é possível representar em todos os "nós" desta estrutura a interação de quatro subsistemas, tendo todos os subsistemas o mesmo número de interações principais com os demais. Estas condições permitem que no processo de agrupamento não haja favorecimento do agrupamento de um subsistema em detrimento do outro, prevalecendo como critério preponderante de agrupamento as condições conservação de cada subsistema.

Na figura 1 está apresentado o octaedro com a alocação dos doze subsistemas. As posições dos subsistemas foram definidas buscando unir em um mesmo nó os subsistemas que possuem maior interação entre si. Mesmo assim alguns subsistemas tiveram que ser conectados a outros com os quais não possuem ligação estreita, o que é aceitável dado que, de modo global, todos os elementos relacionam-se entre si para permitir uma adequada operação da ponte.

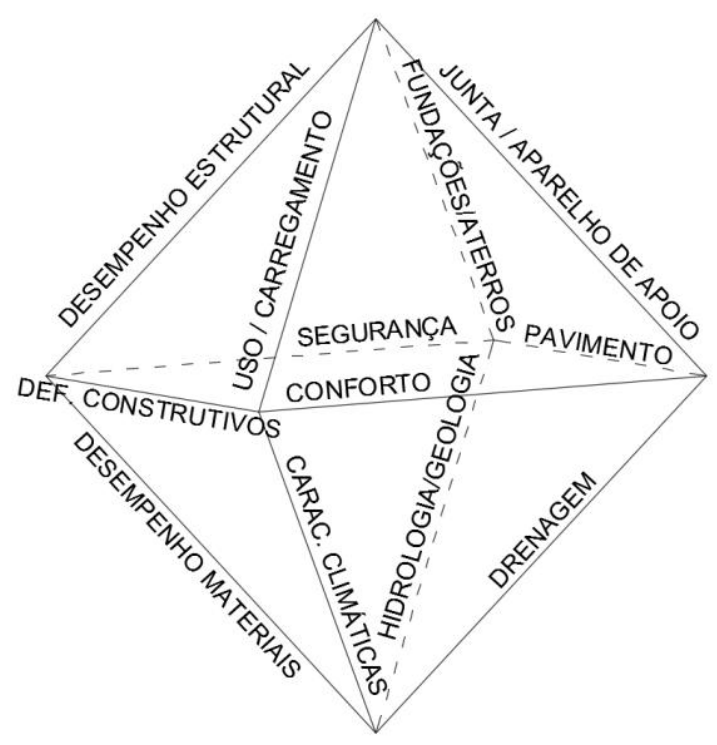

Figura 1: Estrutura que representa as principais interações entre os subsistemas de operação

\subsubsection{Agrupamento dos subsistemas}

O processo de agrupamento, para obtençao da descrição hierárquica da estrutura, é iniciado pelo subsistema com a melhor condição de conservação (menor Índice de Performance) e na sequência são acrescentados um a um os subsistemas restantes, priorizando sempre o que resultar no menor aumento do Índice de Performance do novo grupo formado. O agrupamento deve respeitar as interações principais existentes entre os subsistemas (conexões do octaedro). O Índice de 
DOMINGUES, G. B.; SANTOS FILHO, M. L.; PARCHEN, C. F. A., PROPOSTA DE MODELO DE ANÁLISE DA VULNERABILIDADE DA OPERAÇÃO DE PONTES E VIADUTOS. $3^{\circ}$ Simpósio Paranaense de Patologia das Construções (3 SPPC), artigo 3SPPC1007, pp. $72-83$, 2018. DOI: $10.4322 / 2526-7248.007$

Performance de cada novo grupo formado é dado pela soma dos Índices de Performance dos grupos que o formaram. O processo de agrupamento deve ser continuado até que todos os membros tenham sido adicionados.

\subsubsection{Cenários de deterioração}

Um evento de deterioração, para este modelo, é qualquer ação que danifica um subsistema, ou um componente deste, de modo que ele passa a operar sem o máximo desempenho possível. A simulação de danos na estrutura pode ser feita de duas maneiras: alterando as condições de um ou mais danos que constituem o subsitema, ou alterando diretamente 0 valor do Índice de Performance do subsistema.

\subsection{4 Índice de Vulnerabilidade}

O Índice de Vulnerabilidade (IV), parâmetro que retrata a desproporcionalidade entre o dano que afetou a OAE e as suas consequências, será dado da mesma forma que na Teoria da Vulnerabilidade Estrutural: pela razão entre a Consequência (C) e a Demanda de Dano Relativa (DR) do cenário de falha em análise (equação 2). Dessa forma o Índice de Vulnerabilidade compara as consequências da deterioração de um elemento para a estrutura, com o esforço que foi necessário para causar esse dano, dada as condições atuais da estrutura. A Consequência de um cenário de falha será dada pelo aumento do Índice de Performance geral que a OAE teve em decorrência do dano aplicado:

$$
C=\frac{I P_{\text {deteriorada }}-I P_{\text {atual }}}{I P_{\text {atual }}}
$$

Onde:

$\mathrm{IP}_{\text {deteriorada }}=$ Índice de Performance da ponte com o dano do cenário de falha estudado aplicado;

$\mathrm{IP}_{\text {atual }}=$ Índice de Performance da ponte no momento da inspeção.

A Demanda de Dano Relativa (DR) será dada pela razão entre a demanda de dano do evento de deterioração em estudo e a demanda de dano máxima possível para o elemento afetado (equação 6). A Demanda de Dano (D), ou seja, o esforço necessário para provocar um evento de deterioração, será considerada igual ao aumento do Índice de Performance específico do subsistema afetado diretamente pelo dano. A Demanda de Dano Máxima (Dmáx) é dada quando o subsistema foi completamente deteriorado, ou seja, teve o maior Índice de Performance específico possível, recebendo os maiores valores de K2, K3 e K4.

$$
D_{R}=\frac{D}{D_{\text {máx }}}=\frac{I P_{\text {subs. deteriorado }}-I P_{\text {subsistema }}}{I P_{\text {máx. subs. deteriorado }}-I P_{\text {subsistema }}}
$$

Onde:

$\mathrm{IP}_{\text {subs.deteriorado }}$ I Índice de Performance do subsistema afetado pelo cenário de falha em estudo;

$\mathrm{IP}_{\text {subsistema }}=$ Índice de Performance do subsistema segundo inspeção de campo; 
DOMINGUES, G. B.; SANTOS FILHO, M. L.; PARCHEN, C. F. A., PROPOSTA DE MODELO DE ANÁLISE DA VULNERABILIDADE DA OPERAÇÃO DE PONTES E VIADUTOS. $3^{\circ}$ Simpósio Paranaense de Patologia das Construções (30 SPPC), artigo 3SPPC1007, pp. 72 - 83, 2018. DOI: 10.4322/2526-7248.007

$\mathrm{IP}_{\text {máx.subs.deteriorado }}=$ Índice de Performance do subsistema com a máxima deterioração possível.

\subsection{Exemplo de aplicação}

O modelo foi aplicado em uma ponte com configuração de danos, proveniente da inspeção em campo, apresentada na tabela 7, e Índice de Performance Geral, resultante da soma do valores de dano, igual a 17,16.

Tabela 7: Configuração de danos dos susbsistemas da ponte

\begin{tabular}{|c|c|c|c|c|c|c|c|}
\hline Subsistema & Dano & B & K1 & K2 & K3 & K4 & VD \\
\hline \multirow{4}{*}{$\begin{array}{l}\text { Desempenho } \\
\text { dos materiais }\end{array}$} & Manchas de fogo & 1,00 & 0,50 & 0,50 & 1,50 & 1,00 & 0,38 \\
\hline & Corrosão da armadura & 3,50 & 0,50 & 0,50 & 1,50 & 2,50 & 3,28 \\
\hline & Manchas de umidade & 1,00 & 0,50 & 1,00 & 1,00 & 4,00 & 2,00 \\
\hline & Eflorescências & 1,00 & 0,50 & 0,00 & 1,50 & 1,00 & 0,00 \\
\hline \multirow{2}{*}{$\begin{array}{c}\text { Defeitos } \\
\text { Construtivos } \\
\text { Drenagem }\end{array}$} & $\begin{array}{c}\text { Deficiência de cobrimento } \\
\text { da armadura }\end{array}$ & 2,00 & 0,40 & 0,50 & 1,50 & 2,50 & 1,50 \\
\hline & $\begin{array}{l}\text { Deficiência nas } \\
\text { pingadeiras e buzinotes }\end{array}$ & 2,00 & 0,20 & 2,00 & 2,00 & 2,50 & 4,00 \\
\hline \multirow{2}{*}{ Segurança } & Barreira não padronizada & 2,00 & 0,10 & 2,00 & 2,00 & 1,00 & 0,80 \\
\hline & Sinalização deficiente & 2,00 & 0,10 & 1,00 & 2,00 & 1,00 & 0,40 \\
\hline $\begin{array}{l}\text { Conforto do } \\
\text { usuário }\end{array}$ & $\begin{array}{c}\text { Ausência de passeio em } \\
\text { área urbana }\end{array}$ & 2,00 & 0,20 & 2,00 & 1,50 & 4,00 & 4,80 \\
\hline
\end{tabular}

Para ilustrar a simulação de cenários de falha na OAE, foram aplicadas duas situações de deterioração da ponte, a primeira consiste em uma piora do Sistema de Segurança, e a segunda em uma alteração no Uso e Carregamento Estrutural.

A simulação do cenário de falha que contempla o decaimento das condições de conservação do Sistema de Segurança será feita considerando que as sinalizações vertical e horizontal pioraram substancialmente, e que ambos os guarda-corpos foram rompidos devido a choques de veículos, conforme representado na tabela 8.

Tabela 8: Nova configuração de danos no subsistema de segurança

\begin{tabular}{cccccccc}
\hline Subsistema & Dano & B & K1 & K2 & K3 & K4 & VD \\
\hline \multirow{4}{*}{ Segurança } & Barreira não padronizada & 2,00 & 0,10 & 2,00 & 2,00 & 1,00 & 0,80 \\
& Sinalização deficiente & 2,00 & 0,10 & 2,00 & 2,00 & 2,50 & 2,00 \\
& Transição perigosa & 2,00 & 0,10 & 0,00 & 0,50 & 1,00 & 0,00 \\
& Guarda-corpo danificado & 2,00 & 0,10 & 2,00 & 2,00 & 2,50 & 2,00 \\
\hline
\end{tabular}

Dados os novos Valores de Dano, o Índice de Performance da Segurança passa a ser 4,80, e o Índice de Performance geral da OAE aumenta de 17,16 para 20,76.

Para o cenário de falha compreendido pela alteração no Uso e Carregamento Estrutural foi considerada a modificação das características de tráfego de veículos na região, de modo que passou a existir trânsito de cargas elevadas e frequência alta de carga pesada, conforme a tabela 9.

Assim o Índice de Performance do Uso e Carregamento estrutural passa a ser 13,50, o Índice de Performance geral da OAE passa de 17,16 para 26,16. 
DOMINGUES, G. B.; SANTOS FILHO, M. L.; PARCHEN, C. F. A., PROPOSTA DE MODELO DE ANÁLISE DA VULNERABILIDADE DA OPERAÇÃO DE PONTES E VIADUTOS. $3^{\circ}$ Simpósio Paranaense de Patologia das Construções (3 SPPC), artigo 3SPPC1007, pp. $72-83$, 2018. DOI: $10.4322 / 2526-7248.007$

Tabela 9: Nova configuração de danos no subsistema de uso e carregamento estrutural

\begin{tabular}{cccccccc}
\hline Subsistema & Dano & B & K1 & K2 & K3 & K4 & VD \\
\hline & Trânsito de cargas & 1,00 & 0,60 & 1,50 & 2,00 & 2,50 & 4,50 \\
elevadas & & & & & & \\
Uso / & $\begin{array}{c}\text { Trem-tipo de projeto } \\
\text { Carregamento }\end{array}$ & 3,00 & 0,60 & 0,00 & 0,50 & 1,00 & 0,00 \\
Estrutural & $\begin{array}{c}\text { Frequência alta de carga } \\
\text { pesada }\end{array}$ & 3,00 & 0,60 & 1,00 & 2,00 & 2,50 & 9,00 \\
& $\begin{array}{c}\text { Limitação da capacidade } \\
\text { de carga }\end{array}$ & 5,00 & 0,60 & 0,00 & 0,50 & 1,00 & 0,00 \\
\hline
\end{tabular}

cenário de falha decorrente de alterações na segurança tem Consequência igual a 0,210 (equação 7) e Demanda de Dano Relativa de 0,310 (equação 8), considerando para cálculo da Demanda de Dano Máxima o maior Índice de Performance possível para a Segurança, que obtido considerando o pior comportamento possível dos critérios relacionados. Deste modo, para este cenário de falha o Índice de Vulnerabilidade é 0,677.

$$
\begin{gathered}
C=\frac{20,76-17,16}{17,16}=0,210 \\
D_{R}=\frac{4,80-1,20}{12,80-1,20}=0,310
\end{gathered}
$$

Para o cenário de falha com aumento no Uso e Carregamento Estrutural a Consequência é de 0,524; a Demanda de Dano Relativa é igual a 0,094, e o Índice de Vulnerabilidade é de 5,574.

Comparando os dois cenários apresentados infere-se que o Índice de Vulnerabilidade do segundo é oito vezes maior que o do primeiro cenário, e desse modo observa-se que alterações no carregamento da estrutura, para a OAE em questão, são mais comprometedoras que alterações nos parâmetros de segurança, mesmo este já estando deteriorado. Assim tomando uma situação de gerenciamento de OAEs, em que esteja sendo feita uma análise para investimentos na Ponte, tem-se que é prioritária a destinação de recursos para reforço estrutural caso se tenha uma perspectiva de que o tráfego de veículos pesados aumente no futuro, mesmo estando a OAE, atualmente, respondendo adequadamente ao fluxo de veículos existente.

\section{Conclusão}

O modelo proposto teve um desenvolvimento preliminar, a fim de se investigar a aplicabilidade de uma analogia à Teoria da Vulnerabilidade Estrutural para análise do desempenho funcional de OAEs. Desse modo, o modelo deve ser primeiramente consolidado, aprimorando o desenvolvimento das considerações feitas, e então validado através da aplicação em um grande número de estruturas, para que se possa estudar o comportamento dos resultados obtidos e realizar os ajustes e modificações necessários. 
DOMINGUES, G. B.; SANTOS FILHO, M. L.; PARCHEN, C. F. A., PROPOSTA DE MODELO DE ANÁLISE DA VULNERABILIDADE DA OPERAÇÃO DE PONTES E VIADUTOS. $3^{\circ}$ Simpósio Paranaense de Patologia das Construções (30 SPPC), artigo 3SPPC1007, pp. $72-83$, 2018. DOI: $10.4322 / 2526-7248.007$

A análise proposta permite a identificação não necessariamente dos eventos de deterioração com maior probabilidade de ocorrência, mas sim dos eventos que tem as consequências mais graves. Entretanto pode ser aplicada sobre os cenários de falha identificados uma análise das respectivas probabilidades de ocorrência.

O modelo permite simular o comportamento de uma OAE, dadas as suas condições atuais de conservação, quando for submetida a um tipo de dano geral, como por exemplo, o aumento da agressividade industrial, ou a um dano localizado em algum elemento. Assim é possível fazer considerações tanto sobre ações previsíveis e esperadas, como a degradação natural dos elementos, quanto sobre ações imprevisíveis, tal como o choque de um veículo contra um pilar.

A aplicação de uma análise sob o ponto de vista da vulnerabilidade pode ser um importante parâmetro para tomada de decisão em ferramentas de gestão de OAEs. Ao engenheiro que possui conhecimentos específicos sobre todos os aspectos relacionados à este tipo de estrutura, permite que estes sejam usados para realizar análises amplas, considerando todos os fatores envolvidos. Ao gestor, que possui habilidades de gerenciamento, fornece dados mais concretos para embasar a tomada de decisão. Assim a aplicação do estudo da vulnerabilidade conforme proposto neste trabalho permite uma interação entre esses dois campos, da gestão e da engenharia, permitindo o desenvolvimento de ferramentas de gestão adequadas para tomada de decisão.

\section{Referências}

[1] Agarwal, J.; Blockley, D.; Woodman, N. (2001) Vulnerability Of 3-Dimensional Trusses, Structural Safety, 23: 203-220.

[2] Agarwal, J.; Blockley, D.; Woodman, N. (2003) Vulnerability Of Structural Systems, Structural Safety, 25: 263-286.

[3] Confederação Nacional do Transporte (CNT). (2014) CNT Transporte Atual, 230.

[4] Confederação Nacional do Transporte (CNT). (2017) Pesquisa CNT de rodovias 2017: relatório gerencial.

[5] England, J.; Agarwal, J.; Blockley, D. (2008) The Vulnerability Of Structures To Unforeseen Events, Computers \& Structures, 86: 1042-1051.

[6] Escritório Modelo de Engenharia Civil (EMEA). (2016a) Diretrizes para Inspeção de Obras de Arte Especiais, Universidade Federal do Paraná.

[7] Escritório Modelo de Engenharia Civil (EMEA). (2016b) Obras de Arte Especiais: Manual Técnico, Universidade Federal do Paraná.

[8] Pinto, J.; Blockley, D.; Woodman, N. (2002) The Risk Of Vulnerable Failure, Structural Safety, 24: 107-122. 